\title{
Location Based Generalized Akash Distribution: Properties and Applications
}

\author{
Ramajeyam Tharshan1,2, Pushpakanthie Wijekoon ${ }^{3}$ \\ ${ }^{1}$ Postgraduate Institute of Science, University of Peradeniya, Peradeniya, Sri Lanka \\ ${ }^{2}$ Department of Mathematics and Statistics, University of Jaffna, Jaffna, Sri Lanka \\ ${ }^{3}$ Department of Statistics and Computer Science, University of Peradeniya, Peradeniya, Sri Lanka \\ Email: tharshan10684@gmail.com,pushpaw@pdn.ac.lk
}

How to cite this paper: Tharshan, R. and Wijekoon, P. (2020) Location Based Generalized Akash Distribution: Properties and Applications. Open Journal of Statistics, 10, 163-187.

https://doi.org/10.4236/ojs.2020.102013

Received: February 3, 2020

Accepted: March 31, 2020

Published: April 3, 2020

Copyright (c) 2020 by author(s) and Scientific Research Publishing Inc. This work is licensed under the Creative Commons Attribution International License (CC BY 4.0).

http://creativecommons.org/licenses/by/4.0/

\section{(c) (i) Open Access}

\begin{abstract}
In recent years, several statistical finite mixture models have been proposed to model the lifetime data with heterogeneity. The Lindley distribution has been highlighted by many authors for these types of lifetime data analysis. This paper introduces a new Lindley family distribution called location-based generalized Akash distribution (NGAD) with monotonic increasing and bathtub failure rates. The density function of NGAD is flexible to cover the left-skewed, right-skewed and symmetrical shapes with different tail-weights. Its fundamental structural properties and its ability to provide a suitable statistical model for various types of data sets are studied. The maximum likelihood (ML) method is used to estimate its unknown parameters and the performance of ML estimates are examined by a simulation study. Finally, several real-data sets with different characteristics are used to illustrate its flexibility. It is observed that NGAD provides a better fit than some other existing modified Lindley distributions.
\end{abstract}

\section{Keywords}

Lindley Distribution, Akash Distribution, Mixture Distributions,

Failure Rates, Maximum Likelihood Estimation

\section{Introduction}

Several standard base-line distributions are available to model the lifetime data and the failure rate function is considered as the most crucial factor for these models. Examples of such distributions are exponential, gamma, Weibull, and log-normal distributions which have different capabilities to describe the shapes of the failure rate function. While the exponential distribution has a constant fail- 
ure rate, the gamma and Weibull distributions have both increasing and decreasing failure rates [1]. Researchers in the recent past have an increased interest in modifications of the above-mentioned standard distributions to handle a complicated task for the modeling of lifetime data. The finite mixture models that consist of a weighted sum of the standard or modifications of standard distributions are popular to handle the complexity by heterogeneity in a lifetime data analysis. Each finite mixture failure rate may have various characteristics, such as monotonically increasing or decreasing, non-monotonic, constant, and bathtub shapes, and they may cover various tail-heaviness of a data set. We can measure the tailheaviness of a data set by the excess kurtosis $(E K)$ that is defined as $\gamma-3$, where $\gamma$ is the kurtosis of the data set. The $E K>0$ is called as heavy-tailed (Leptokurtic) and $E K<0$ is called light-tailed (Platykurtic) distributions. Some notables are mentioned as, Hussaini, Dayian, and Adham (2000) introduced a twocomponent Gompertz lifetime model [2]; Marin, Berna, and Wiper (2005) obtained the Weibull mixture distributions [3]; Balakrishnan, Leiva, Sanhueza, and Cabrera (2009) proposed a mixture of inverse Gaussian distribution [4]; Erisoglu, Erisoglu, and Erol (2011) introduced a mixture model of two different distributions approach [5]; among others. However, more finite mixtures are complicated in their mathematical forms.

Another classical finite mixture model under the Bayesian framework is the Lindley distribution (LD) which is a finite mixture of two non-identical distributions, exponential and gamma distributions. It was introduced by Lindley (1958) [6] [7] having the density function:

$$
f_{\Lambda}(\lambda)=\frac{\theta^{2}}{1+\theta}(1+\lambda) \mathrm{e}^{-\theta \lambda} ; \lambda>0, \theta>0,
$$

where $\theta$ is the shape parameter, and $\lambda$ is the respective random variable. Equation (1) presents two-component mixture of exponential $(\theta)$ and gamma $(2, \theta)$ distributions with the mixing proportion $p=\frac{\theta}{1+\theta}$. The statistical properties of LD have discussed by Ghitany, Atieh, and Nadarajah (2008) and showed that the Lindley distribution is more flexible and provides a better fit than the exponential distribution for lifetime data [8].

A considerable number of modifications of $\mathrm{LD}$ have been proposed by researchers with actual mixing components of $\mathrm{LD}$ based on the two-component mixture of exponential $(\theta)$ and gamma ( $2, \theta)$ with different mixing proportions. Some notables are mentioned as, Shanker and Sharma (2013) proposed a two-parameter Lindley distribution [9]; Shanker and Mishra (2013) obtained the Quasi Lindley distribution [10]; Shanker (2015) introduced the Shanker distribution [11]; and Shanker, Shukla, and Tekie (2017) introduced a three-parameter Lindley distribution [12], and abbreviations of these distributions are TwPLD, QLD, SD, and ThPLD, respectively.

To increase more flexibility in this line of developments, Monsef (2016) [13] introduced a new three-parameter family Lindley distribution called Lindley with 
location distribution (LwLD) by adding the location parameter. The density function of this distribution is given by:

$$
\begin{aligned}
& f_{\Lambda}(\lambda)=\frac{\theta^{2}}{\theta+\alpha}(1+\alpha(\lambda-\beta)) \mathrm{e}^{-\theta(\lambda-\beta)} ; \\
& \lambda>\beta>0, \theta>0,1+\alpha \lambda>0, \alpha+\theta>0,
\end{aligned}
$$

where $\theta$ and $\alpha$ are shape parameters and $\beta$ is a location parameter. Equation (2) presents two-component mixture of an exponential $(\theta, \beta)$ and gamma ( $2, \theta, \beta)$ distributions with the mixing proportion $p=\frac{\theta}{\theta+\alpha}$.

On the other hand, Shanker (2015) [14] has obtained Akash distribution (AD) as an alternative to Lindley distribution with density function:

$$
f_{\Lambda}(\lambda)=\frac{\theta^{3}}{\theta^{2}+2}\left(1+\lambda^{2}\right) \mathrm{e}^{-\theta \lambda} ; \lambda>0 ; \theta>0,
$$

where $\theta$ is a shape parameter. Equation (3) shows two-component mixture of exponential $(\theta)$ and gamma $(3, \theta)$ with mixing proportion $\frac{\theta^{2}}{\theta^{2}+2}$.

Table 1 summarizes the above-mentioned existing Lindley family distributions' names, abbreviations, parameters, shapes of the failure rate function, and references.

In many lifetime distributions, the location parameter may be assumed to be zero. However, the location parameter is an important parameter that provides an estimate of the failure-free period, that is the earliest time at which a failure may be observed. When we include the location parameter in a distribution, it changes the starting point of the distribution. For example, time to achieve pain relief in a patient after who has applied with a treatment method does not start from the value of zero. Then, in this application, the location parameter of the

\begin{tabular}{|c|c|c|c|c|c|c|}
\hline \multirow[t]{2}{*}{ Distribution } & \multirow[t]{2}{*}{ Abbreviation } & \multicolumn{3}{|c|}{ Parameters } & \multirow[t]{2}{*}{ Failure rate } & \multirow[t]{2}{*}{ References } \\
\hline & & scale & shape & location & & \\
\hline Lindley & $\mathrm{LD}$ & - & $\theta$ & - & increasing & Lindley (1958) [7] \\
\hline Two-parameter Lindley & TwPLD & - & $\theta, \alpha$ & - & increasing & Shanker et al. (2013) [9] \\
\hline Quasi Lindley & QLD & $\theta$ & $\alpha$ & - & increasing & Shanker et al. (2013) [10] \\
\hline Shanker & SD & - & $\theta$ & - & increasing & Shanker (2015) [11] \\
\hline Akash & $\mathrm{AD}$ & - & $\theta$ & - & increasing & Shanker (2015) [14] \\
\hline $\begin{array}{l}\text { Lindley with location } \\
\text { distribution }\end{array}$ & LwLD & - & $\theta, \alpha$ & $\beta$ & increasing & Monsef (2016) [13] \\
\hline Three-parameter Lindley & ThPLD & - & $\theta, \alpha, \beta$ & - & increasing & Shanker et al. (2017) [12] \\
\hline & & & & & \& decreasing & \\
\hline
\end{tabular}
relevant distribution cannot be taken as zero.

Table 1. Distributions' names, abbreviations, parameters, shapes of the failure rate function, and references. 
In this paper, we introduce and study a new three-parameter Lindley family distribution with location parameter as a modification of $\mathrm{AD}$. The new distribution will be called as location based generalized Akash distribution (NGAD). The NGAD is a two-component mixture of exponential and gamma distributions which generalizes the AD. Further, a simulation study will be done to study the performance of the ML method in the parameter estimation of the NGAD.

The remaining part of this paper is organized as follows: In Section 2, we introduce the NGAD with its density and distribution functions. We present the statistical and reliability properties of NGAD in Section 3 and Section 4, respectively. The size-biased form of the NGAD is discussed in Section 5. Further, the estimation of the parameters of NGAD by using the ML method is discussed in Section 6. Finally, a simulation study is done to study the performance of the maximum likelihood estimators for NGAD, and real-world applications are used to illustrate its flexibility with the above-mentioned existing modified Lindley distributions.

\section{Location Based Generalized Akash Distribution}

In this section, we introduce the location based generalized Akash distribution with its probability density function (pdf) and cumulative distribution function (cdf). Note that the Akash distribution (3) contains only one shape parameter. To develop the new distribution first, we define a new three-parameter Lindley family distribution by including the location parameter $\beta$ as a finite mixture of exponential $(\theta, \beta)$ and gamma $(3, \theta, \beta)$ with mixing proportion $p=\frac{\theta^{2}}{\theta^{2}+\eta}$ under the Bayesian framework, as follows:

$$
f_{\Lambda}(\lambda ; \theta, \beta, \eta)=p f_{1}(\lambda ; \theta, \beta)+(1-p) f_{2}(\lambda ; 3, \theta, \beta),
$$

where, $\theta$ and $\eta$ are shape parameters and

$$
f_{1}(\lambda ; \theta, \beta)=\theta \mathrm{e}^{-\theta(\lambda-\beta)} \text {, and } f_{2}(\lambda ; 3, \theta, \beta)=\frac{\theta^{3}(\lambda-\beta)^{2}}{2} ; \lambda>\beta>0 ; \theta>0 .
$$

Then, the pdf of the NGAD with parameters $\theta, \beta$ and $\eta$ is defined by:

$$
\begin{aligned}
& f_{\Lambda}(\lambda ; \theta, \beta, \eta)=\frac{\theta^{3}}{2\left(\theta^{2}+\eta\right)}\left(2+\eta(\lambda-\beta)^{2}\right) \mathrm{e}^{-\theta(\lambda-\beta)} ; \\
& \lambda>\beta>0, \theta>0, \theta^{2}>-\eta .
\end{aligned}
$$

The range of the parameters is based on the log-likelihood function. Then, NGAD has one additional shape parameter and the location parameter compared to $\mathrm{AD}$ 's parameter. Plots in Figure 1 have drawn by fixing 2 parameters and changing the $3^{\text {rd }}$ parameter. Figure 1 shows how the shape parameters $\theta$ and $\eta$ affect the shape of the NGAD and how the location parameter affects the starting point of the distribution. Here, we may observe that the pdf of NGAD is either a unimodal or a monotonic decreasing function. Further, when we fix other 2 parameters, 


$$
\beta=1.5 \eta=1.25
$$

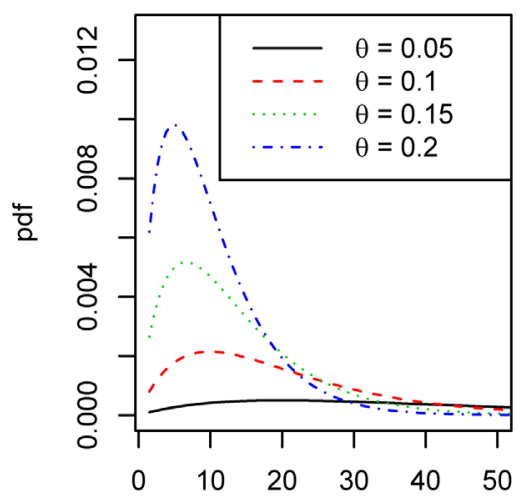

(a)

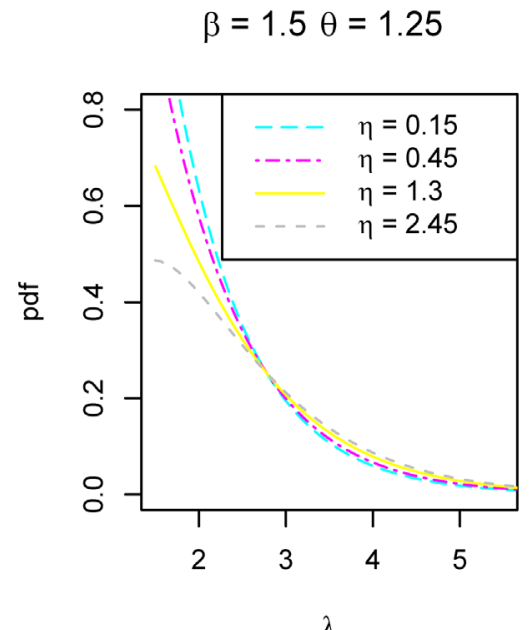

(d)

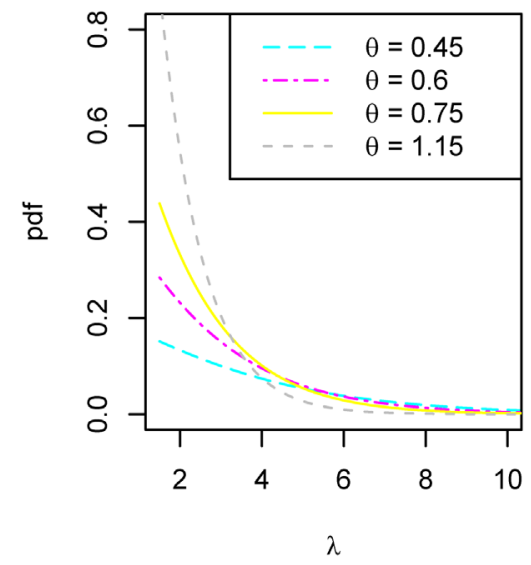

(b)

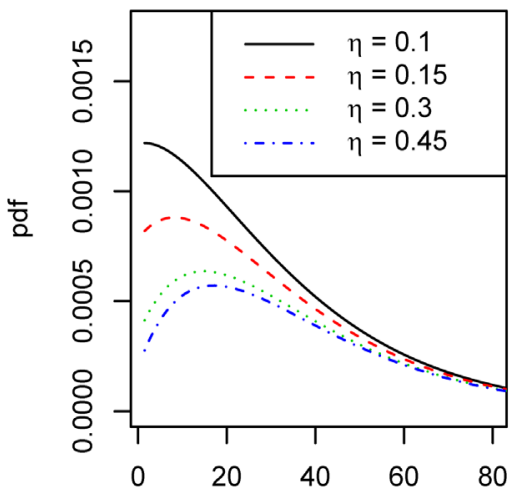

(c)

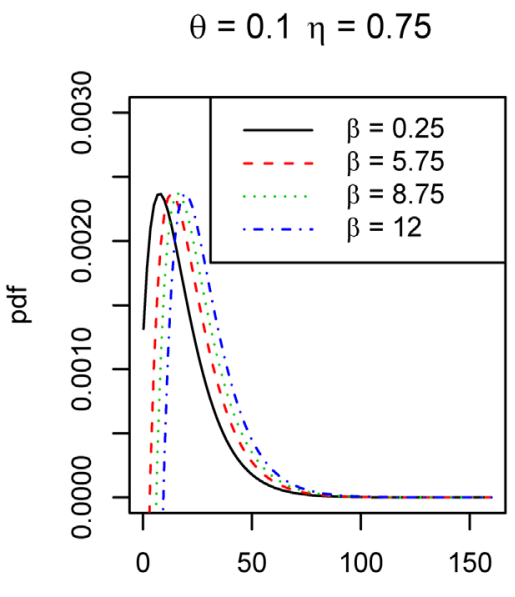

$(\mathrm{e})$

Figure 1 . The probability density of NGAD at different parameter values. (a) and (b): $\beta$, and $\eta$ are fixed, and $\theta$ values are changed; (c) and (d): $\theta$, and $\beta$ are fixed, and $\eta$ values are changed; (e): $\theta$, and $\eta$ are fixed, and $\beta$ values are changed.

from Figure 1(a) and Figure 1(b), the shapes tend to be flatten as $\theta$ decreases and from Figure 1(c) and Figure 1(d), the shapes tend to be flattened as $\eta$ increases in both cases.

The mode value in the unimodal case can be derived directly from the first derivative of Equation (4), and given as:

$$
f^{\prime}(\lambda)=\frac{\theta^{3} \mathrm{e}^{-\theta(\lambda-\beta)}}{2\left(\theta^{2}+\eta\right)}\left(2 \eta(\lambda-\beta)-\theta\left(2+\eta(\lambda-\beta)^{2}\right)\right) .
$$

Then, $f^{\prime}(\lambda)=0$ gives the mode value:

$$
\lambda_{0}=\frac{\eta(\beta \theta+1)+\sqrt{\eta\left(\eta-2 \theta^{2}\right)}}{\eta \theta} ; \eta>2 \theta^{2} \text { and } \lambda_{0}>\beta \text {. }
$$

The corresponding cumulative distribution function of NGAD is given by: 


$$
F_{\Lambda}(\lambda)=1-\left(1+\frac{\eta\left(2 \theta \lambda+(\theta \lambda)^{2}-\beta \theta(2(1+\theta \lambda)-\beta \theta)\right)}{2\left(\theta^{2}+\eta\right)}\right) \mathrm{e}^{-\theta(\lambda-\beta)},
$$

where $\lambda>\beta>0, \theta>0, \theta^{2}>-\eta$.

Note that when $\eta=2, \beta=0$, the NGAD reduces to the $\mathrm{AD}$ with parameter $\theta$.

\section{Statistical Properties}

Here, some important statistical properties of the NGAD are derived such as $I^{\text {th }}$ moments and related measures, moment generating and characteristic functions, and quantile function.

\subsection{Moments and Related Measures}

Some basic important characteristics of the distribution such as central tendency, dispersion, skewness, kurtosis, and index of dispersion can be studied by using the moments. The following theorem gives the $r^{\text {th }}$ moment about the origin.

Theorem 1. The $r^{\text {th }}$ moment about the origin of the NGAD is given by:

$$
\begin{aligned}
\mu_{r}^{\prime}= & \frac{1}{2\left(\theta^{2}+\eta\right) \theta^{r}}\left(r \mathrm{e}^{\theta \beta} \Gamma(r, \theta \beta)\left(2 \theta^{2}+\eta\left((r+1)(r+2)-2 \beta \theta(r+1)+\beta^{2} \theta^{2}\right)\right)\right. \\
& \left.+(\theta \beta)^{r}\left(2 \theta^{2}+\eta((r+1)(r+2)-r \beta \theta)\right)\right) .
\end{aligned}
$$

\section{Proof.}

$$
\begin{aligned}
\mu_{r}^{\prime}= & \int_{\beta}^{\infty} \lambda^{r} \frac{\theta^{3}}{2\left(\theta^{2}+\eta\right)}\left(2+\eta(\lambda-\beta)^{2}\right) \mathrm{e}^{-\theta(\lambda-\beta)} \mathrm{d} \lambda \\
= & \frac{\theta^{3} \mathrm{e}^{\theta \beta}}{2\left(\theta^{2}+\eta\right)}\left(2 \int_{\beta}^{\infty} \lambda^{r} \mathrm{e}^{-\theta \lambda} \mathrm{d} \lambda+\eta \int_{\beta}^{\infty} \lambda^{r}(\lambda-\beta)^{2} \mathrm{e}^{-\theta \lambda} \mathrm{d} \lambda\right) \\
= & \frac{\theta^{3} \mathrm{e}^{\theta \beta}}{2\left(\theta^{2}+\eta\right)}\left(\frac{2}{\theta^{r+1}} \Gamma(r+1, \theta \beta)+\eta\left(\frac{1}{\theta^{r+3}} \Gamma(r+3, \theta \beta)\right.\right. \\
& \left.\left.-\frac{2 \beta}{\theta^{r+2}} \Gamma(r+2, \theta \beta)+\frac{\beta^{2}}{\theta^{r+1}} \Gamma(r+1, \theta \beta)\right)\right) \\
= & \frac{1}{2\left(\theta^{2}+\eta\right) \theta^{2}}\left(r \Gamma(r, \theta \beta) \mathrm{e}^{\theta \beta}\left(2 \theta^{2}+\eta\left((r+1)(r+2)-2 \beta \theta(r+1)+\beta^{2} \theta^{2}\right)\right)\right. \\
& \left.+(\theta \beta)^{r}\left(2 \theta^{2}+\eta((r+1)(r+2)-r \beta \theta)\right)\right) \\
= & \frac{1}{2\left(\theta^{2}+\eta\right) \theta^{r}}\left(r \mathrm{e}^{\theta \beta} \Gamma(r, \theta \beta)\left(2 \theta^{2}+\eta\left((r+1)(r+2)-2 \beta \theta(r+1)+\beta^{2} \theta^{2}\right)\right)\right. \\
& \left.+(\theta \beta)^{r}\left(2 \theta^{2}+\eta((r+1)(r+2)-r \beta \theta)\right)\right) .
\end{aligned}
$$

The first four moments about the origin are derived by substituting $r=1,2,3$, and 4 in Equation (6) as: 


$$
\begin{gathered}
\mu_{1}^{\prime}=\frac{\theta^{2}(1+\theta \beta)+\eta(3+\theta \beta)}{\left(\theta^{2}+\eta\right) \theta}=\mu, \\
\mu_{2}^{\prime}=\frac{\theta^{2}(2+\theta \beta(2+\theta \beta))+\eta(12+\theta \beta(6+\theta \beta))}{\left(\theta^{2}+\eta\right) \theta^{2}}, \\
\mu_{3}^{\prime}=\frac{\theta^{2}(6+\theta \beta(6+\theta \beta(3+\theta \beta)))+\eta(60+\theta \beta(36+\theta \beta(9+\theta \beta)))}{\left(\theta^{2}+\eta\right) \theta^{3}}, \text { and } \\
\mu_{4}^{\prime}=\frac{\theta^{2}(24+\theta \beta(24+\theta \beta(12+\theta \beta(4+\theta \beta)))+\eta(360+\theta \beta(240+\theta \beta(72+\theta \beta(12+\theta \beta))))}{\left(\theta^{2}+\eta\right) \theta^{4}},
\end{gathered}
$$

respectively. Then, the $r^{\text {th }}$-order moments about the mean can be obtained by using the relationship between moments about the mean and moments about the origin; i.e.

$$
\mu_{r}=E\left[(\Lambda-\mu)^{r}\right]=\sum_{i=0}^{r}\left(\begin{array}{l}
r \\
i
\end{array}\right)(-1)^{r-i} \mu_{i}^{\prime} \mu^{r-i}
$$

Therefore, $\mu_{2}=\sigma^{2}, \mu_{3}$, and $\mu_{4}$ are obtained as:

$$
\begin{gathered}
\mu_{2}=-\mu^{2}+\mu_{2}^{\prime}=\frac{\theta^{4}+\eta\left(8 \theta^{2}+3 \eta\right)}{\left(\theta^{2}+\eta\right)^{2} \theta^{2}}, \\
\mu_{3}=2 \mu^{3}-3 \mu_{2}^{\prime} \mu+\mu_{3}^{\prime}=\frac{2 \theta^{6}+\eta\left(30 \theta^{4}+\eta\left(18 \theta^{2}+6 \eta\right)\right)}{\left(\theta^{2}+\eta\right)^{3} \theta^{3}}, \text { and } \\
\mu_{4}=-3 \mu^{4}+6 \mu_{2}^{\prime} \mu^{2}-4 \mu_{3}^{\prime} \mu+\mu_{4}^{\prime} \\
=\frac{9 \theta^{8}+\eta\left(192 \theta^{6}+\eta\left(306 \theta^{4}+\eta\left(216 \theta^{2}+45 \eta\right)\right)\right)}{\left(\theta^{2}+\eta\right)^{4} \theta^{4}},
\end{gathered}
$$

respectively. Then, the the coefficient of variation $(c . V)$, measures of skewness $\left(\gamma_{1}\right)$, kurtosis $\left(\gamma_{2}\right)$ and the Index of dispersion/Fano factor $\left(\gamma_{3}\right)$ of the NGAD can be derived as:

$$
\begin{gathered}
c . v=\frac{\left(\mu_{2}\right)^{1 / 2}}{\mu_{1}^{\prime}}=\frac{\sqrt{\theta^{4}+\eta\left(8 \theta^{2}+3 \eta\right)}}{\theta^{2}(1+\theta \beta)+\eta(3+\theta \beta)}, \\
\gamma_{1}=\frac{\mu_{3}}{\left(\mu_{2}\right)^{3 / 2}}=\frac{2 \theta^{6}+\eta\left(30 \theta^{4}+\eta\left(18 \theta^{2}+6 \eta\right)\right)}{\left(\theta^{4}+\eta\left(8 \theta^{2}+3 \eta\right)\right)^{\frac{3}{2}}}, \\
\gamma_{2}=\frac{\mu_{4}}{\mu_{2}^{2}}=\frac{9 \theta^{8}+\eta\left(192 \theta^{6}+\eta\left(306 \theta^{4}+\eta\left(216 \theta^{2}+45 \eta\right)\right)\right)}{\left(\theta^{4}+\eta\left(8 \theta^{2}+3 \eta\right)\right)^{2}}, \text { and } \\
\gamma_{3}=\frac{\mu_{2}}{\mu_{1}^{\prime}}=\frac{\theta^{4}+\eta\left(8 \theta^{2}+3 \eta\right)}{\theta\left(\theta^{2}+\eta\right)\left(\theta^{2}(1+\theta \beta)+\eta(3+\theta \beta)\right)},
\end{gathered}
$$


respectively. Table 2 shows the skewness and kurtosis values at different parameter value settings. We can observe that for a fixed $\theta$ or $\eta$, skewness and kurtosis show a mixed behaviour while other parameter increases.

\subsection{Moment Generating and Characteristic Function}

The moment generating function (mgf) and the characteristic function (cf) are directly associated with a probability distribution's characteristics. Further, these can be used to generate the moments of a distribution. The following theorem provides the moment generating function of the NGAD.

Theorem 2. The moment generating function say $M_{\Lambda}(t)$ of the $N G A D$ is given as follows.

$$
\begin{aligned}
M_{\Lambda}(t)= & \frac{\theta^{3} \mathrm{e}^{\beta t}}{2\left(\theta^{2}+\eta\right)(\theta-t)^{3}}\left(2(\theta-t)^{2}+\eta(2+\beta(\theta-t)(2+\beta(\theta-t)\right. \\
& -2(1+\beta(\theta-t))+\beta(\theta-t)))) .
\end{aligned}
$$

Proof.

$$
\begin{aligned}
M_{\Lambda}(t)= & E\left(\mathrm{e}^{t \lambda}\right)=\int_{\beta}^{\infty} \mathrm{e}^{t \lambda} \frac{\theta^{3}}{2\left(\theta^{2}+\eta\right)}\left(2+\eta(\lambda-\beta)^{2}\right) \mathrm{e}^{-\theta(\lambda-\beta)} \mathrm{d} \lambda \\
= & \frac{\theta^{3} \mathrm{e}^{\theta \beta}}{2\left(\theta^{2}+\eta\right)}\left(2 \int_{\beta}^{\infty} \mathrm{e}^{-\lambda(\theta-t)} \mathrm{d} \lambda+\eta \int_{\beta}^{\infty} \mathrm{e}^{-\lambda(\theta-t)}(\lambda-\beta)^{2} \mathrm{~d} \lambda\right) \\
= & \frac{\theta^{3} \mathrm{e}^{\theta \beta}}{2\left(\theta^{2}+\eta\right)}\left(\frac{2 \mathrm{e}^{-\beta(\theta-t)}}{\theta-t}+\eta\left(\frac{\Gamma(3, \beta(\theta-t))}{(\theta-t)^{3}}\right.\right. \\
& \left.\left.-\frac{2 \beta \Gamma(2, \beta(\theta-t))}{(\theta-t)^{2}}+\frac{\beta^{2} \mathrm{e}^{-\beta(\theta-t)}}{\theta-t}\right)\right) \\
= & \frac{\theta^{3} \mathrm{e}^{\beta t}}{2\left(\theta^{2}+\eta\right)(\theta-t)^{3}}\left(2(\theta-t)^{2}+\eta(2+\beta(\theta-t)(2+\beta(\theta-t)\right. \\
& -2(1+\beta(\theta-t))+\beta(\theta-t))))
\end{aligned}
$$

Table 2. Skewness(Kurtosis) at different values of $\theta$ and $\eta$.

\begin{tabular}{ccccccc}
\hline$\downarrow \theta \rightarrow \eta$ & 0.01 & 0.10 & 0.45 & 0.95 & 1.45 & 1.95 \\
\hline 0.03 & $1.092(4.825)$ & $1.144(4.978)$ & $1.152(4.995)$ & $1.153(4.997)$ & $1.154(4.998)$ & $1.155(4.999)$ \\
0.05 & $1.089(4.727)$ & $1.129(4.939)$ & $1.148(4.985)$ & $1.151(4.993)$ & $1.152(4.995)$ & $1.153(4.996)$ \\
0.10 & $1.347(5.333)$ & $1.089(4.812)$ & $1.132(4.945)$ & $1.143(4.973)$ & $1.147(4.982)$ & $1.148(4.987)$ \\
0.20 & $1.883(7.842)$ & $1.130(4.768)$ & $1.092(4.826)$ & $1.116(4.903)$ & $1.127(4.933)$ & $1.133(4.949)$ \\
0.45 & $2.123(9.790)$ & $1.618(6.415)$ & $1.147(4.798)$ & $1.083(4.733)$ & $1.082(4.772)$ & $1.088(4.807)$ \\
0.70 & $2.084(9.621)$ & $1.948(8.264)$ & $1.376(5.432)$ & $1.171(4.846)$ & $1.110(4.741)$ & $1.089(4.727)$ \\
0.95 & $2.054(9.415)$ & $2.082(9.282)$ & $1.614(6.397)$ & $1.330(5.278)$ & $1.211(4.937)$ & $1.151(4.806)$ \\
1.20 & $2.036(9.285)$ & $2.120(9.689)$ & $1.802(7.361)$ & $1.501(5.902)$ & $1.344(5.326)$ & $1.254(5.051)$ \\
1.45 & $2.026(9.205)$ & $2.122(9.793)$ & $1.934(8.167)$ & $1.655(6.589)$ & $1.483(5.831)$ & $1.373(5.420)$ \\
\hline
\end{tabular}


Similarly, the characteristic function say, $\psi(t)$ of the NGAD can be derived as follows:

$$
\begin{aligned}
\psi_{\Lambda}(t)= & E\left(\mathrm{e}^{i t \lambda}\right) \\
= & \frac{\theta^{3} \mathrm{e}^{\beta i t}}{2\left(\theta^{2}+\eta\right)(\theta-i t)^{3}}\left(2(\theta-i t)^{2}+\eta(2+\beta(\theta-i t)(2+\beta(\theta-i t)\right. \\
& -2(1+\beta(\theta-i t))+\beta(\theta-i t)))) .
\end{aligned}
$$

\subsection{Quantile Function}

The quantile function is useful for quantile estimates and random number generation. The quantile function of NGAD can be derived by solving $F(\lambda)=u, 0<u<1$. Then, the $u^{t h}$ quantile function of NGAD is derived as:

$$
\begin{aligned}
F\left(\lambda_{u}\right) & =1-\left(1+\frac{\eta\left(2 \theta \lambda_{u}+\left(\theta \lambda_{u}\right)^{2}-\beta \theta\left(2\left(1+\theta \lambda_{u}\right)-\beta \theta\right)\right)}{2\left(\theta^{2}+\eta\right)}\right) \mathrm{e}^{-\theta\left(\lambda_{u}-\beta\right)}=u . \\
\Rightarrow & \left(2\left(\theta^{2}+\eta\right)+\eta\left(2 \theta \lambda_{u}+\left(\theta \lambda_{u}\right)^{2}-\beta \theta\left(2\left(1+\theta \lambda_{u}\right)-\beta \theta\right)\right)\right) \mathrm{e}^{-\theta\left(\lambda_{u}-\beta\right)} \\
& -2\left(\theta^{2}+\eta\right)(1-u)=0 .
\end{aligned}
$$

Equation (9) is not a closed-form. However, the $u^{\text {th }}$ quantile can be estimated and random varieties from NGAD can be generated by using the numerical methods.

By substituting $u=0.25,0.5$ and 0.75 in Equation (9), the first three quartiles can be derived by solving the following equations, respectively.

$$
\begin{aligned}
& \left(2\left(\theta^{2}+\eta\right)+\eta\left(2 \theta \lambda_{0.25}+\left(\theta \lambda_{0.25}\right)^{2}-\beta \theta\left(2\left(1+\theta \lambda_{0.25}\right)-\beta \theta\right)\right)\right) \mathrm{e}^{-\theta\left(\lambda_{0.25}-\beta\right)} \\
& -2\left(\theta^{2}+\eta\right) 0.75=0, \\
& \left(2\left(\theta^{2}+\eta\right)+\eta\left(2 \theta \lambda_{0.5}+\left(\theta \lambda_{0.5}\right)^{2}-\beta \theta\left(2\left(1+\theta \lambda_{0.5}\right)-\beta \theta\right)\right)\right) \mathrm{e}^{-\theta\left(\lambda_{0.5}-\beta\right)} \text { and } \\
& -2\left(\theta^{2}+\eta\right) 0.5=0, \\
& \left(2\left(\theta^{2}+\eta\right)+\eta\left(2 \theta \lambda_{0.75}+\left(\theta \lambda_{0.75}\right)^{2}-\beta \theta\left(2\left(1+\theta \lambda_{0.75}\right)-\beta \theta\right)\right)\right) \mathrm{e}^{-\theta\left(\lambda_{0.75}-\beta\right)} \\
& -2\left(\theta^{2}+\eta\right) 0.25=0 .
\end{aligned}
$$

\section{Reliability, Inequality and Entropy Measures}

In this study, we derive important reliability measures of NGAD such as survival function/reliability function $S(\lambda)$, hazard rate function/failure rate function $h(\lambda)$, reversed hazard rate function $r(\lambda)$, cumulative hazard rate function $H(\lambda)$, mean residual life function $m(\lambda)$, and inequality measures, namely Lorenz curve $L(\lambda)$, and Benferroni curve $B(\lambda)$. Further, we obtain Renyi entropy measure associated to NGAD. 


\subsection{Survival and Hazard Rate Functions}

The survival function and hazard rate function are crucial functions to specify a survival distribution. The survival function is the probability of surviving up to a point $\lambda$. Then, the survival function of NGAD is defined as:

$$
\begin{aligned}
S(\lambda) & =p(\Lambda>\lambda)=1-F(\lambda) \\
& =\left(1+\frac{\eta\left(2 \theta \lambda+(\theta \lambda)^{2}-\beta \theta(2(1+\theta \lambda)-\beta \theta)\right)}{2\left(\theta^{2}+\eta\right)}\right) \mathrm{e}^{-\theta(\lambda-\beta)} ; \lambda>\beta .
\end{aligned}
$$

It is clear that, $S(\beta)=1$ and $\lim _{\lambda \rightarrow \infty} S(\lambda)=0$.

The hazard rate function (hrf) is the instantaneous failure rate. It is widely used to describe the lifetime. The hrf of the NGAD is defined as:

$$
\begin{aligned}
h(\lambda) & =\lim _{\Delta \lambda \rightarrow 0} \frac{P(\lambda<\Lambda<\lambda+\Delta \lambda \mid \Lambda>\lambda)}{\Delta \lambda}=\frac{f(\lambda)}{S(\lambda)} \\
& =\frac{\theta^{3}\left(2+\eta(\lambda-\beta)^{2}\right)}{2\left(\theta^{2}+\eta\right)+\eta(\theta \lambda(2+\theta \lambda)-\beta \theta(2(1+\theta \lambda)-\beta \theta))} .
\end{aligned}
$$

Note that, $h(\beta)=\frac{\theta^{3}}{\theta^{2}+\eta}$ and $\lim _{\lambda \rightarrow \infty} h(\lambda)=\theta$.

Figure 2 presents the possible shapes of the proposed distribution's hazard rate function at different shape parameter values. Then, the proposed model has the capability to model the bathtub and monotonic increasing failure rate shapes while the $\mathrm{AD}$ has only a monotonic increasing failure rate.

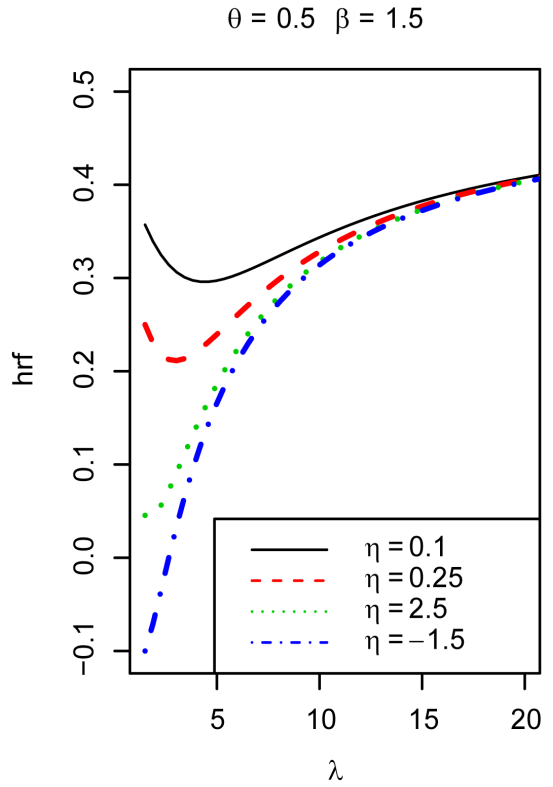

(a)

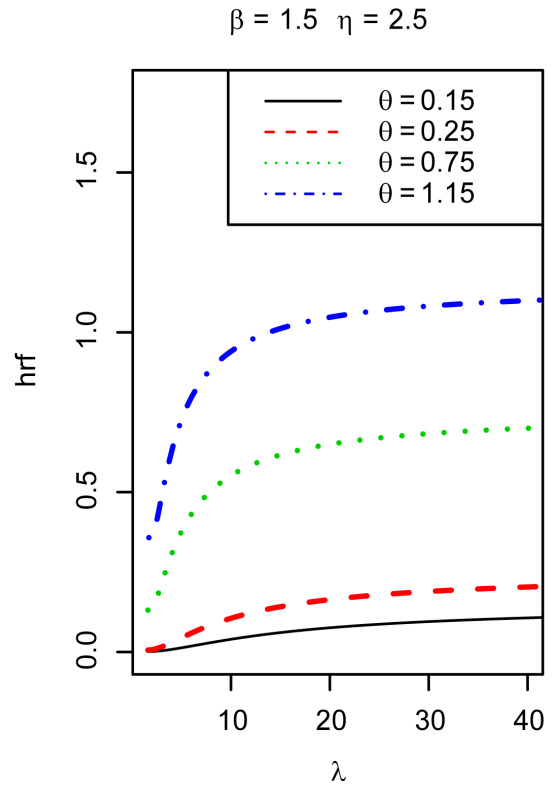

(b)

Figure 2. The hazard rate function of NGAD at different parameter values. (a) $\theta$ and $\beta$ are fixed, and $\eta$ values are changed, (b) $\beta$ and $\eta$ are fixed, and $\theta$ values are changed. 
The corresponding reversed hazard rate function of NGAD is defined as:

$$
\begin{aligned}
r(\lambda) & =\lim _{\Delta \lambda \rightarrow 0} \frac{P(\lambda<\Lambda<\lambda+\Delta \lambda \mid \Lambda<\lambda)}{\Delta \lambda} \\
& =\frac{\theta^{3}\left(2+\eta(\lambda-\beta)^{2}\right) \mathrm{e}^{-\theta(\lambda-\beta)}}{2\left(\theta^{2}+\eta\right)-\left(2 \theta^{2}+\eta(2+\theta \lambda(2+\theta \lambda)-\beta \theta(2(1+\theta \lambda)-\beta \theta))\right) \mathrm{e}^{-\theta(\lambda-\beta)}},
\end{aligned}
$$

and the cumulative hazard rate function of NGAD is defined as:

$$
\begin{aligned}
H(\lambda) & =\int_{\beta}^{\lambda} h(t) \mathrm{d} t=-\ln [S(\lambda)] \\
& =-\ln \left(1+\frac{\eta(\theta \lambda(2+\theta \lambda)-\beta \theta(2(1+\theta \lambda)-\beta \theta))}{2\left(\theta^{2}+\eta\right)}\right)+\theta(\lambda-\beta) .
\end{aligned}
$$

\subsection{Mean Residual Life Function}

The residual life function is defined as the remaining lifetime of a unit of age $\lambda>\beta$ until the time of failure. The following theorem derives the mean residual life function of NGAD.

Theorem 3. The mean residual life function of $N G A D$ is given by:

$$
m(\lambda)=\frac{2 \theta^{2}+\eta(6-\beta \theta(4-\beta \theta)+\theta \lambda(4-2 \beta \theta+\theta \lambda))}{\theta\left(2\left(\theta^{2}+\eta\right)+\eta(\theta \lambda(2+\theta \lambda)-\beta \theta(2(1+\theta \lambda)-\beta \theta))\right)} ; \lambda>\beta .
$$

Proof. $m(\lambda)=\frac{1}{1-F(\lambda)} \int_{\lambda}^{\infty} t f(t) \mathrm{d} t-\lambda$.

Now, consider the integrals separately as follows:

$$
\begin{aligned}
& \int_{\lambda}^{\infty} t f(t) \mathrm{d} t \\
& =\int_{\lambda}^{\infty} t \frac{\theta^{3}}{2\left(\theta^{2}+\eta\right)}\left(2+\eta(t-\beta)^{2}\right) \mathrm{e}^{-\theta(t-\beta)} \mathrm{d} t \\
& =\frac{\theta^{3} \mathrm{e}^{\theta \beta}}{2\left(\theta^{2}+\eta\right)}\left(2 \int_{\lambda}^{\infty} t \mathrm{e}^{-\theta t} \mathrm{~d} t+\eta\left(\int_{\lambda}^{\infty} t^{3} \mathrm{e}^{-\theta t} \mathrm{~d} t-2 \beta \int_{\lambda}^{\infty} t^{2} \mathrm{e}^{-\theta t} \mathrm{~d} t+\beta^{2} \int_{\lambda}^{\infty} t \mathrm{e}^{-\theta t} \mathrm{~d} t\right)\right) \\
& =\frac{\theta^{3} \mathrm{e}^{\theta \beta}}{2\left(\theta^{2}+\eta\right)}\left(\frac{2 \Gamma(2, \theta \lambda)}{\theta^{2}}+\eta\left(\frac{\Gamma(4, \theta \lambda)}{\theta^{4}}-\frac{2 \beta \Gamma(3, \theta \lambda)}{\theta^{3}}+\frac{\beta^{2} \Gamma(2, \theta \lambda)}{\theta^{2}}\right)\right) \\
& =\frac{\mathrm{e}^{-\theta(\lambda-\beta)}}{2\left(\theta^{2}+\eta\right) \theta}\left(2 \theta^{2}(1+\theta \lambda)+A\right)
\end{aligned}
$$

where, $A=\eta(6-\beta \theta(4-\beta \theta)+\theta \lambda(6-\beta \theta(4-\beta \theta)+\theta \lambda(3-2 \beta \theta+\theta \lambda)))$. Therefore,

$$
\begin{aligned}
m(\lambda) & =\frac{2 \theta^{2}(1+\theta \lambda)+A}{\theta\left(2\left(\theta^{2}+\eta\right)+\eta(\theta \lambda(2+\theta \lambda)-\beta \theta(2(1+\theta \lambda)-\beta \theta))\right)}-\lambda \\
& =\frac{2 \theta^{2}+\eta(6-\beta \theta(4-\beta \theta)+\theta \lambda(4-2 \beta \theta+\theta \lambda))}{\theta\left(2\left(\theta^{2}+\eta\right)+\eta(\theta \lambda(2+\theta \lambda)-\beta \theta(2(1+\theta \lambda)-\beta \theta))\right)} .
\end{aligned}
$$

Then, Equation (14) satisfies the following properties 


$$
m(\lambda) \geq 0, m(\beta)=\frac{\theta^{2}+3 \eta}{\theta\left(\theta^{2}+\eta\right)}, \quad \text { and } \lim _{\lambda \rightarrow \infty} m(\lambda)=\frac{1}{\theta}
$$

\subsection{Lorenz and Bonferroni Curves}

The Lorenz and Bonferroni curves were formulated to measure the income inequality. They are widely used in economics, reliability, demography, medicine, and insurance. The following theorem derives the Lorenz curve for NGLD.

Theorem 4. The Lorenz curve is defined for FPGLD as

$$
L(F(\lambda))=1-\frac{\int_{\lambda}^{\infty} x f(x) \mathrm{d} x}{\mu}=1-\frac{\mathrm{e}^{-\theta(\lambda-\beta)}\left(2 \theta^{2}(1+\theta \lambda)+B\right)}{2\left(\theta^{2}(1+\theta \beta)+\eta(3+\theta \beta)\right)},
$$

where, $B=\eta\left(6+\theta \lambda\left(6+3 \theta \lambda+(\theta \lambda)^{2}\right)-\beta \theta(4+\theta \lambda(4+2 \theta \lambda-\beta \theta)-\beta \theta)\right)$

Proof. $L(F(\lambda))=1-\frac{\int_{\lambda}^{\infty} x f(x) \mathrm{d} x}{\mu}$.

Note that

$$
\begin{aligned}
\int_{\lambda}^{\infty} x f(x) \mathrm{d} x \\
=\int_{\lambda}^{\infty} x \frac{\theta^{3}}{2\left(\theta^{2}+\eta\right)}\left(2+\eta(x-\beta)^{2}\right) \mathrm{e}^{-\theta(x-\beta)} \mathrm{d} x \\
=\frac{\theta^{3} \mathrm{e}^{\theta \beta}}{2\left(\theta^{2}+\eta\right)}\left(2 \int_{\lambda}^{\infty} x \mathrm{e}^{-\theta x} \mathrm{~d} x+\eta \int_{\lambda}^{\infty} x(x-\beta)^{2} \mathrm{e}^{-\theta x} \mathrm{~d} x\right) \\
=\frac{\theta^{3} \mathrm{e}^{\theta \beta}}{2\left(\theta^{2}+\eta\right)}\left(2 \int_{\lambda}^{\infty} x \mathrm{e}^{-\theta x} \mathrm{~d} x+\eta\left(\int_{\lambda}^{\infty} x^{3} \mathrm{e}^{-\theta x} \mathrm{~d} x-2 \beta \int_{\lambda}^{\infty} x^{2} \mathrm{e}^{-\theta x} \mathrm{~d} x+\beta^{2} \int_{\lambda}^{\infty} x \mathrm{e}^{-\theta x} \mathrm{~d} x\right)\right) \\
=\frac{\theta^{3} \mathrm{e}^{\theta \beta}}{2\left(\theta^{2}+\eta\right)}\left(\frac{2 \Gamma(2, \theta \lambda)}{\theta^{2}}+\eta\left(\frac{\Gamma(4, \theta \lambda)}{\theta^{4}}-\frac{2 \beta \Gamma(3, \theta \lambda)}{\theta^{3}}+\frac{\beta^{2} \Gamma(2, \theta \lambda)}{\theta^{2}}\right)\right) \\
=\frac{\mathrm{e}^{-\theta(\lambda-\beta)}}{2\left(\theta^{2}+\eta\right) \theta}\left(2 \theta^{2}(1+\theta \lambda)+\eta\left(6+\theta \lambda\left(6+3 \theta \lambda+(\theta \lambda)^{2}\right)\right.\right. \\
\quad-\beta \theta(4+\theta \lambda(4+2 \theta \lambda-\beta \theta)-\beta \theta))) .
\end{aligned}
$$

Therefore,

$$
L(F(\lambda))=1-\frac{\mathrm{e}^{-\theta(\lambda-\beta)}\left(2 \theta^{2}(1+\theta \lambda)+B\right)}{2\left(\theta^{2}(1+\theta \beta)+\eta(3+\theta \beta)\right)},
$$

and the corresponding Bonferroni curve for the NGAD is defined as:

$$
\begin{aligned}
B(F(\lambda)) & =\frac{L(F(\lambda))}{F(\lambda)} \\
& =\frac{2\left(\theta^{2}+\eta\right)\left(2\left(\theta^{2}(1+\theta \beta)+\eta(3+\theta \beta)\right)-\left(2 \theta^{2}(1+\theta \lambda)+C\right) \mathrm{e}^{-\theta(\lambda-\beta)}\right)}{2\left(\theta^{2}(1+\theta \beta)+\eta(3+\theta \beta)\right)\left(2\left(\theta^{2}+\eta\right)-\left(2 \theta^{2}+D\right) \mathrm{e}^{-\theta(\lambda-\beta)}\right)},
\end{aligned}
$$

where, 


$$
\begin{gathered}
C=\eta\left(6+\theta \lambda\left(6+3 \theta \lambda+(\theta \lambda)^{2}\right)-\beta \theta(4+\theta \lambda(4+2 \theta \lambda-\beta \theta)-\beta \theta)\right) \text { and } \\
D=\eta(2+\theta \lambda(2+\theta \lambda)-\beta \theta(2(1+\theta \lambda)-\beta \theta)) .
\end{gathered}
$$

\subsection{Renyi Entropy}

The Renyi entropy is a measure of variation of uncertainty measure of a distribution say $H_{\gamma}(\lambda)$ [15]. It is an extension of Shannon entropy [16] and widley used in information theory. The following theorem derives the Renyi entropy associated with NGAD.

Theorem 5. The Renyi entropy of the NGAD is given by:

$$
\begin{aligned}
H_{\gamma}(\lambda) & =\frac{1}{1-\gamma} \ln \int_{\beta}^{\infty}(f(\lambda))^{\gamma} \mathrm{d} \lambda \\
& =\frac{1}{1-\gamma} \ln \left(\frac{\theta^{3 \gamma}}{\left(\theta^{2}+\eta\right)^{\gamma}} \sum_{k=0}^{\gamma} \frac{\left(\begin{array}{l}
\gamma \\
k
\end{array}\right) \eta^{k} \Gamma(2 k+1)}{2^{k}(\gamma \theta)^{2 k+1}}\right) ; \gamma \geq 0, \gamma \neq 1 .
\end{aligned}
$$

Proof.

$$
\begin{aligned}
H_{\gamma}(\lambda) & =\frac{1}{1-\gamma} \ln \int_{\beta}^{\infty}(f(\lambda))^{\gamma} \mathrm{d} \lambda \\
& =\frac{1}{1-\gamma} \ln \left(\frac{\theta^{3 \gamma}}{\left(\theta^{2}+\eta\right)^{\gamma}} \int_{\beta}^{\infty} \sum_{k=0}^{\gamma}\left(\begin{array}{l}
\gamma \\
k
\end{array}\right)\left(\frac{\eta(\lambda-\beta)^{2}}{2}\right)^{k} \mathrm{e}^{-\gamma \theta(\lambda-\beta)} \mathrm{d} \lambda\right) \\
& =\frac{1}{1-\gamma} \ln \left(\frac{\theta^{3 \gamma}}{\left(\theta^{2}+\eta\right)^{\gamma}} \sum_{k=0}^{\gamma}\left(\begin{array}{l}
\gamma \\
k
\end{array}\right)\left(\frac{\eta}{2}\right)^{k} \frac{1}{(\gamma \theta)^{2 k+1}} \int_{\beta}^{\infty} y^{2 k} \mathrm{e}^{-y} \mathrm{~d} y\right) ; y=\gamma \theta(\lambda-\beta) \\
& =\frac{1}{1-\gamma} \ln \left(\frac{\theta^{3 \gamma}}{\left(\theta^{2}+\eta\right)^{\gamma}} \sum_{k=0}^{\gamma} \frac{\left(\begin{array}{l}
\gamma \\
k
\end{array}\right) \eta^{k} \Gamma(2 k+1)}{2^{k}(\gamma \theta)^{2 k+1}}\right) .
\end{aligned}
$$

\section{The Size-Biased of NGAD}

When a recording of observations with an unequal chance, the weighted distributions are used significantly. This provides more flexibility to the standard distributions incorporating sampling probabilities which are proportional to a non-negative weighted function $w(\lambda)$. The applications of the weighted distributions in reliability, biomedical and ecological sciences have been studied by Patil and Rao (1978) [17]. The weighted random variable $\Lambda_{w}$ of NGAD is defined as:

$$
f_{\Lambda_{w}}(\lambda)=\frac{w(\lambda) f(\lambda)}{E(w(\lambda))} ; \lambda>\beta
$$

where $E(w(\lambda))=\int_{\beta}^{\infty} w(\lambda) f(\lambda) \mathrm{d} \lambda ; 0<E(w(\lambda))<\infty$.

When $w(\lambda)=\lambda^{\gamma}, \gamma>0$, the resulting distribution is called size-biased ver- 
sion of NGAD with order $\gamma$, and is defined as:

$$
f_{\Lambda_{s}}^{\gamma}(\lambda)=\frac{\lambda^{\gamma} f(\lambda)}{E\left(\lambda^{\gamma}\right)} ; \lambda>\beta, \gamma>0,
$$

where $\Lambda_{s}$ is the respective random variable.

The following theorem gives the density function for the sized-biased version of NGAD.

Theorem 6. The density function for $r^{\text {th }}$ order sized-biased form of NGAD is derived as:

$$
\begin{aligned}
& f_{\Lambda_{s}}^{\gamma}(\lambda)=\frac{\lambda^{\gamma} \theta^{\gamma+3}\left(2+\eta(\lambda-\beta)^{2}\right) \mathrm{e}^{-\theta \lambda}}{\Gamma(\gamma+1, \theta \beta)\left(2 \theta^{2}+E\right)+\eta \mathrm{e}^{-\theta \beta}(\theta \beta)^{\gamma+1}(\gamma+2-\theta \beta)} \\
& \lambda>\beta, \gamma>0,
\end{aligned}
$$

where $E=\eta\left((\gamma+1)(\gamma+2)-2 \theta \beta(\gamma+1)+\beta^{2} \theta^{2}\right)$

Proof. $f_{\Lambda_{s}}^{\gamma}(\lambda)=\frac{\lambda^{\gamma} f(\lambda)}{E\left(\lambda^{\gamma}\right)}$.

Note that

$$
\begin{aligned}
E\left(\lambda^{\gamma}\right) & =\int_{\beta}^{\infty} \lambda^{\gamma} f(\lambda) \mathrm{d} \lambda \\
& =\int_{\beta}^{\infty} \lambda^{\gamma} \frac{\theta^{3}}{2\left(\theta^{2}+\eta\right)}\left(2+\eta(\lambda-\beta)^{2}\right) \mathrm{e}^{-\theta(\lambda-\beta)} \mathrm{d} \lambda \\
& =\frac{\theta^{3} \mathrm{e}^{\theta \beta}}{2\left(\theta^{2}+\eta\right)}\left(2 \int_{\beta}^{\infty} \lambda^{\gamma} \mathrm{e}^{-\theta \lambda} \mathrm{d} \lambda+\int_{\beta}^{\infty} \lambda^{\gamma}(\lambda-\beta)^{2} \mathrm{e}^{-\theta \lambda} \mathrm{d} \lambda\right) \\
& =\frac{\theta^{3} \mathrm{e}^{\theta \beta}}{2\left(\theta^{2}+\eta\right)}\left(\frac{\Gamma(\gamma+1, \theta \beta)}{\theta^{\gamma+1}}+\eta\left(\frac{\Gamma(\gamma+3, \theta \beta)}{\theta^{\gamma+3}}\right.\right. \\
\left.\left.-\frac{2 \beta \Gamma(\gamma+2, \theta \beta)}{\theta^{\gamma+2}}+\frac{\beta^{2} \Gamma(\gamma+1, \theta \beta)}{\theta^{\gamma+1}}\right)\right) & \mathrm{e}^{\theta \beta}\left(\Gamma ( \gamma + 1 , \theta \beta ) \left(2 \theta^{2}+\eta((\gamma+1)(\gamma+2)\right.\right. \\
2\left(\theta^{2}+\eta\right) \theta^{\gamma} & \left.\left.\left.-2 \beta \theta(\gamma+1)+(\beta \theta)^{2}\right)\right)+\eta(\theta \beta)^{\gamma+1} \mathrm{e}^{-\theta \beta}(\gamma+2-\theta \beta)\right) .
\end{aligned}
$$

Therefore,

$$
f_{\Lambda_{s}^{\gamma}}^{\gamma}(\lambda)=\frac{\lambda^{\gamma} \theta^{\gamma+3}\left(2+\eta(\lambda-\beta)^{2}\right) \mathrm{e}^{-\theta \lambda}}{\Gamma(\gamma+1, \theta \beta)\left(2 \theta^{2}+E\right)+\eta \mathrm{e}^{-\theta \beta}(\theta \beta)^{\gamma+1}(\gamma+2-\theta \beta)} .
$$

By substituting $\gamma=1$ in Equation (19), the length-biased probability density function can be obtained as:

$$
\frac{\lambda \theta^{4}\left(2+\eta(\lambda-\beta)^{2}\right) \mathrm{e}^{-\theta(\lambda-\beta)}}{(1+\theta \beta)\left(2 \theta^{2}+\eta\left(6-4 \theta \beta+(\beta \theta)^{2}\right)\right)+\eta(\theta \beta)^{2}(3-\theta \beta)} ; \lambda>\beta, \gamma>0 .
$$




\section{Parameter Estimation and Inference}

In this section, the method of ML is introduced to estimate the parameters of NGAD. Further, the confidence intervals for the unknown parameters are derived.

Let $\lambda_{1}, \lambda_{2}, \cdots, \lambda_{n}$ be identically and independently distributed random variables from NGAD with the likelihood function of the $i^{\text {th }}$ sample value $\lambda_{i}$ as:

$$
L\left(\theta, \beta, \eta \mid \lambda_{i}\right)=\frac{\theta^{3}}{2\left(\theta^{2}+\eta\right)}\left(2+\eta\left(\lambda_{i}-\beta\right)^{2}\right) \mathrm{e}^{-\theta\left(\lambda_{i}-\beta\right)} .
$$

Hence, the $\log$-likelihood function $l=\ln (L)$ is given by:

$$
l=3 n \ln \theta-\sum_{i=1}^{n} \theta\left(\lambda_{i}-\beta\right)+\sum_{i=1}^{n} \ln \left(2+\eta\left(\lambda_{i}-\beta\right)^{2}\right)-n \ln \left(2\left(\theta^{2}+\eta\right)\right) .
$$

Then, the ML estimators of $\theta, \beta$, and $\eta$, abbreviated as $\hat{\theta}, \hat{\beta}$, and $\hat{\eta}$ can be derived by equating the partial derivatives of the $I$ with respect to each parameter to zero. Then, the systems of equations are:

$$
\begin{aligned}
& \frac{\partial l}{\partial \theta}=\frac{3 n}{\theta}-\sum_{i=1}^{n}\left(\lambda_{i}-\beta\right)-\frac{2 n \theta}{\theta^{2}+\eta}, \frac{\partial l}{\partial \beta}=n \theta-\sum_{i=1}^{n} \frac{2 \eta\left(\lambda_{i}-\beta\right)}{2+\eta\left(\lambda_{i}-\beta\right)^{2}}, \\
& \text { and } \frac{\partial l}{\partial \eta}=\sum_{i=1}^{n} \frac{\left(\lambda_{i}-\beta\right)^{2}}{2+\eta\left(\lambda_{i}-\beta\right)^{2}}-\frac{n}{\theta^{2}+\eta} .
\end{aligned}
$$

The second partial derivatives of the $l$ are:

$$
\begin{aligned}
& \frac{\partial^{2} l}{\partial \theta^{2}}=\frac{-3 n}{\theta^{2}}-\frac{2 n\left(\eta-\theta^{2}\right)}{\left(\theta^{2}+\eta\right)^{2}}, \frac{\partial^{2} l}{\partial \theta \partial \beta}=n, \frac{\partial^{2} l}{\partial \theta \partial \eta}=\frac{2 n \theta}{\left(\theta^{2}+\eta\right)^{2}}, \\
& \frac{\partial^{2} l}{\partial \beta^{2}}=2 \eta \sum_{i=1}^{n} \frac{2-\eta\left(\lambda_{i}-\beta\right)^{2}}{\left(2+\eta\left(\lambda_{i}-\beta\right)^{2}\right)^{2}}, \frac{\partial^{2} l}{\partial \beta \partial \eta}=-\sum_{i=1}^{n} \frac{4\left(\lambda_{i}-\beta\right)}{\left(2+\eta\left(\lambda_{i}-\beta\right)^{2}\right)^{2}}, \\
& \text { and } \frac{\partial^{2} l}{\partial \eta^{2}}=\frac{n}{\left(\theta^{2}+\eta\right)^{2}}-\sum_{i=1}^{n} \frac{\left(\lambda_{i}-\beta\right)^{4}}{\left(2+\eta\left(\lambda_{i}-\beta\right)^{2}\right)^{2}} .
\end{aligned}
$$

By the asymptotic theory, the estimators are asymptotically normal 3-variate with mean $(\theta, \beta, \eta)$ and the observed information matrix:

$$
I(\theta, \beta, \eta)=\left(\begin{array}{ccc}
-\frac{\partial^{2} l}{\partial \theta^{2}} & -\frac{\partial^{2} l}{\partial \theta \partial \beta} & -\frac{\partial^{2} l}{\partial \theta \partial \eta} \\
-\frac{\partial^{2} l}{\partial \beta \partial \theta} & -\frac{\partial^{2} l}{\partial \beta^{2}} & -\frac{\partial^{2} l}{\partial \beta \partial \eta} \\
-\frac{\partial^{2} l}{\partial \eta \partial \theta} & -\frac{\partial^{2} l}{\partial \eta \partial \beta} & -\frac{\partial^{2} l}{\partial \eta^{2}}
\end{array}\right)
$$

at $\theta=\hat{\theta}, \beta=\hat{\beta}$, and $\eta=\hat{\eta}$. By the asymptotic theory, the estimates are approximately multivariate normal. Therefore, the $(1-a) 100 \%$ confidence interval for the parameters $\theta, \beta$, and $\eta$ are given by

$$
\hat{\theta} \pm z_{a / 2} \sqrt{\operatorname{var}(\hat{\theta})}, \quad \hat{\beta} \pm z_{a / 2} \sqrt{\operatorname{var}(\hat{\beta})}, \quad \hat{\eta} \pm z_{a / 2} \sqrt{\operatorname{var}(\hat{\eta})},
$$

wherein, the $\operatorname{var}(\hat{\theta}), \operatorname{var}(\hat{\beta})$, and $\operatorname{var}(\hat{\eta})$ are the variance of $\hat{\theta}, \hat{\beta}$, and $\hat{\eta}$, respectively, and can be derived by diagonal elements of $I^{-1}(\theta, \beta, \eta)$ and $z_{a / 2}$ is the critical value at a level of significance. 


\section{Applications}

In this section, we examine the performance of the parameter estimates of NGAD by ML method and asymptotic theory respect to sample size $n$. Here, we perform a simulation study, and further, we illustrate the flexibility of the NGAD over the LD, QLD, TwPLD, LwLD, SD, AD, and ThPLD by using several real data sets.

\subsection{Simulation Study}

In this subsection, we present the simulation study results to examine the performance of the estimations by the ML method that proposed in Section 6. Equation (9) is used to carry out the simulation study by generating random samples from NGAD, where $u$ is assumed to follow uniform distribution $(0,1)$. The replicates are 1000 in all the simulations with sample sizes $40,100,160$, and 220 . The combinations of parameter values are set at $(\theta=0.2, \beta=2.5, \eta=1.25)$ to represent the unimodel case and at $(\theta=0.5, \beta=1.5, \eta=0.25)$ to represent the monotonic decreasing case. The average bias and mean square error (MSE) of $\hat{\theta}, \hat{\beta}$ and $\hat{\eta}$ of the parameters $\theta, \beta$ and $\eta$ are computed as follows:

1) The average biases are:

$$
\frac{\sum_{i=1}^{1000}(\hat{\theta}-\theta)}{1000}, \frac{\sum_{i=1}^{1000}(\hat{\beta}-\beta)}{1000}, \frac{\sum_{i=1}^{1000}(\hat{\eta}-\eta)}{1000} .
$$

2) The average MSEs are:

$$
\frac{\sum_{i=1}^{1000}(\hat{\theta}-\theta)^{2}}{1000}, \frac{\sum_{i=1}^{1000}(\hat{\beta}-\beta)^{2}}{1000}, \frac{\sum_{i=1}^{1000}(\hat{\eta}-\eta)^{2}}{1000} .
$$

Table 3 summarizes average bias and MSE values of the estimates by the ML method. The average bias values of parameter estimates are considerably small and the corresponding average MSE values decrease when sample size increases. Then, this verifies the asymptotic property of all the estimates.

\subsection{Real Data Applications}

This subsection is considered to show the flexibility of NGAD over some other existing Lindley family distributions by fitting these to several real data sets. The Akaike Information Criterion (AIC), Bayesian Information Criterion (BIC) and Kolmogorov-Smirnov Statistics (K-S Statistics) are utilized to compare the performance of LD, SD, AD, TwPLD, QLD, LwLD, and ThPLD. The ML method was used to estimate the unknown parameters. The first three real data sets that were considered for the goodness of fit of distributions are given below:

Data set 1: The following data set represents the tensile strength, measured in $\mathrm{GPa}$, of 69 carbon fibres tested under tension at gauge lengths of $20 \mathrm{~mm}$ and reported by Bader and Priest (1982) [18].

$1.312,1.314,1.479,1.552,1.700,1.803,1.861,1.865,1.944,1.958,1.966,1.997$, 2.006, 2.021, 2.027, 2.055, 2.063, 2.098, 2.140, 2.179, 2.224, 2.240, 2.253, 2.270, $2.272,2.274,2.301,2.301,2.359,2.382,2.382,2.426,2.434,2.435,2.478,2.490$, $2.511,2.514,2.535,2.554,2.566,2.570,2.586,2.629,2.633,2.642,2.648,2.684$, 
2.697, 2.726, 2.770, 2.773, 2.800, 2.809, 2.818, 2.821, 2.848, 2.880, 2.954, 3.012, $3.067,3.084,3.090,3.096,3.128,3.233,3.433,3.585,3.585$.

Data set 2: The following data set reported by Lawless (1982) [1] that arose in tests on endurance of deep groove ball bearing. The data are the number of million revolutions before failure for each of the 23 ball bearing in the life tests.

$17.88,28.92$, 33, 41.52, 42.12, 45.6, 48.8, 51.84, 51.96, 54.12, 55.56, 67.8, 68.44, $68.64,68.88,84.12,93.12,98.64,105.12,105.84,127.92,128.04,173.4$.

Data set 3: The following data set represents the tree circumferences in Marshall, Minnesota and reported by Shakil, Kibria, and Singh (2010) [19].

$1.8,1.8,1.9,2.4,3.1,3.4,3.7,3.7,3.8,3.9,4.0,4.1,4.9,5.1,5.1,5.2,5.3,5.5,8.3$, 13.7 .

Some of the statistical measures for data set 1, 2 and 3 are given in Table 4.

The fitted density plot of each distribution is shown in Figure 3 for data set 1, 2 , and 3 separately. Each fitted density compares with the empirical histogram of the real data sets. Here, we may observe that the fitted density for the NGAD shows a closer fit with the empirical distributions. The AIC, BIC, and K-S statistics with critical values for NGAD, LwLD, TwPLD, QLD, ThPLD, AD, LD, and SD are shown in Table S1 (Appendix). Based on minimum AIC, BIC values and significant results by K-S statistics, the NGAD provides a better fit than all other distributions for light-tailed distributions $(E K<0)$ as well as heavy-tailed distributions $(E K>0)$. Further, it is noted that the LwLD performs better than NGAD for the data set with a considerably high positive $E K$ value.

Then, similarly we have fitted NGAD and LwLD to several real-data sets (Appendix) having different $E K$ values and compare their performances. Table 5 summarizes the results of the goodness of fit test for these data sets. It is clear that NGAD may perform better than LwLD for considerably small $E K$ values.

Table 3. Simulation results for average bias and MSE values of NGAD.

\begin{tabular}{ccccc}
\hline \multirow{2}{*}{ Parameter } & \multicolumn{3}{c}{ Average MSE (bias) } \\
\cline { 2 - 4 } & $n=40$ & $n=100$ & $n=160$ & $n=220$ \\
\hline$\theta=0.2$ & 0.006952 & 0.006849 & 0.006763 & 0.006298 \\
$\beta=2.5$ & $(0.029578)$ & $(0.025475)$ & $(0.021125)$ & $(0.019797)$ \\
& 2.274852 & 1.035007 & 0.996790 & 0.899319 \\
$\eta=1.25$ & $(0.510524)$ & $(0.233261)$ & $(0.201401)$ & $(0.173923)$ \\
& 13.811090 & 12.060685 & 7.910364 & 7.140838 \\
& $(1.448722)$ & $(1.343749)$ & $(1.192976)$ & $(1.168940)$ \\
\cline { 2 - 4 }$\beta=0.5$ & $n=40$ & $n=100$ & $n=160$ & $n=220$ \\
& 0.003798 & 0.000793 & 0.000361 & 0.000356 \\
& $(0.017002)$ & $(0.004542)$ & $(0.003929)$ & $(0.002167)$ \\
& 1.699948 & 0.258165 & 0.162436 & 0.120519 \\
& $(0.336223)$ & $(0.018714)$ & $(0.007087)$ & $(0.003035)$ \\
& 17.132331 & 10.118250 & 4.588242 & 4.077955 \\
& $(2.236709)$ & $(1.846210)$ & $(1.514328)$ & $(1.209076)$ \\
\hline
\end{tabular}


Table 4. Statistical measures for data set 1,2 , and 3.

\begin{tabular}{ccccccc}
\hline Data & Sample size & Minimum value & Mean & Median & Skewness & $E K$ \\
\hline Data 1 & 69 & 1.312 & 2.451 & 2.478 & -0.028 & -0.059 \\
Data 2 & 23 & 17.880 & 72.230 & 67.800 & 0.941 & 0.488 \\
Data 3 & 20 & 1.800 & 4.535 & 3.950 & 2.173 & 5.353 \\
\hline
\end{tabular}

Table 5. AICs, and K-S statistics of the NGAD and LwLD for different data sets with various $E K$ values.

\begin{tabular}{|c|c|c|c|c|c|c|}
\hline Data & Distribution & Sample size & Skewness & $E K$ & AIC & $\mathrm{K}-\mathrm{S}$ statistics \\
\hline \multirow[t]{2}{*}{ Data4 } & NGAD & 34 & 0.219 & -0.843 & 47.147 & 0.097 \\
\hline & LwLD & & & & 50.324 & 0.116 \\
\hline \multirow[t]{2}{*}{ Data5 } & NGAD & 31 & 0.405 & -0.713 & 213.769 & 0.103 \\
\hline & LwLD & & & & 215.115 & 0.106 \\
\hline \multirow[t]{2}{*}{ Data6 } & NGAD & 66 & -0.131 & 0.223 & 204.602 & 0.188 \\
\hline & LwLD & & & & 218.816 & 0.222 \\
\hline \multirow[t]{2}{*}{ Data7 } & NGAD & 63 & -0.900 & 0.924 & 80.828 & 0.257 \\
\hline & LwLD & & & & 94.758 & 0.277 \\
\hline \multirow[t]{2}{*}{ Data8 } & NGAD & 100 & 0.372 & 1.120 & 954.899 & 0.172 \\
\hline & LwLD & & & & 980.661 & 0.218 \\
\hline \multirow[t]{2}{*}{ Data9 } & NGAD & 202 & 1.175 & 1.365 & 1915.144 & 0.091 \\
\hline & LwLD & & & & 1902.719 & 0.069 \\
\hline \multirow[t]{2}{*}{ Data10 } & NGAD & 72 & 1.342 & 1.991 & 220.760 & 0.179 \\
\hline & LwLD & & & & 199.551 & 0.141 \\
\hline \multirow[t]{2}{*}{ Data11 } & NGAD & 59 & 1.608 & 2.256 & 388.848 & 0.134 \\
\hline & LwLD & & & & 384.510 & 0.110 \\
\hline \multirow[t]{2}{*}{ Data12 } & NGAD & 72 & 1.473 & 2.890 & 668.821 & 0.336 \\
\hline & LwLD & & & & 531.213 & 0.186 \\
\hline \multirow[t]{2}{*}{ Data13 } & NGAD & 20 & 1.720 & 2.924 & 38.025 & 0.143 \\
\hline & LwLD & & & & 36.987 & 0.121 \\
\hline
\end{tabular}
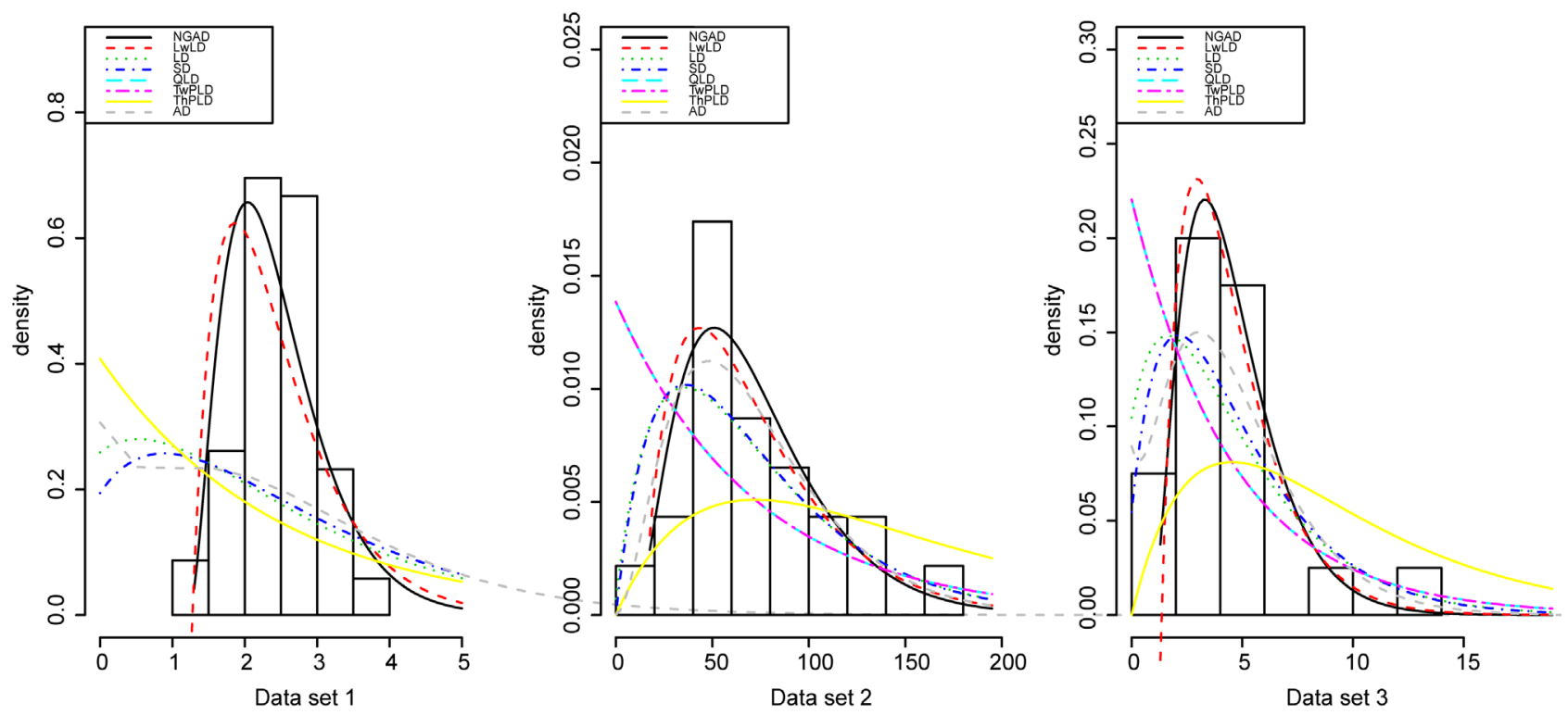

Figure 3. Empirical histograms with fitted densities of distributions. 


\section{Conclusion}

In this study, a new generalized Akash distribution has been introduced by incorporating the location parameter to improve the flexibility of the failure rate function. Then, its structural properties including parameter estimation have been discussed. This new distribution yields a more flexible density and failure rate shapes. Further, it has the capability to model the bathtub and monotonic increasing failure rate shapes. The simulation study indicates that the ML method performs well in the estimation of the unknown parameters for NGAD. To illustrate the theoretical findings, the real-world applications were used, and the results reveal that the NGAD is superior over $\mathrm{AD}$, Lindley distribution (LD) and some other existing modified LDs that have been developed based on exponential and gamma mixtures with different mixing proportions. Based on the special characteristics, the proposed model may attract wider applications in reliability, mortality, actuarial, ecological sciences, among others.

\section{Acknowledgements}

We thank the Postgraduate Institute of Science, University of Peradeniya, Sri Lan$\mathrm{ka}$ for providing all facilities to do this research.

\section{Conflicts of Interest}

The authors declare no conflicts of interest regarding the publication of this paper.

\section{References}

[1] Lawless, J.F. (1982) Statistical Models and Methods for Lifetime Data. John Wiley and Sons, New York.

[2] Al-Hussaini, E.K., Al-Dayian, G.R. and Adham, S.A. (2000) On Finite Mixture of Two-Component Gompertz Lifetime Model. Journal of Statistical Computation and Simulation, 67, 1-20. https://doi.org/10.1080/00949650008812033

[3] Marin, J., Rodriguez-Bernal, M. and Wiper, M. (2005) Using Weibull Mixture Distributions to Model Heterogeneous Survival Data. Communications in Statistics-Simulation and Computation, 34, 673-684. https://doi.org/10.1081/SAC-200068372

[4] Balakrishnan, N., Leiva, V., Sanhueza, A. and Cabrera, E. (2009) Mixture Inverse Gaussian Distributions and Its Transformations, Moments and Applications. Statistics, 43, 91-104. https://doi.org/10.1080/02331880701829948

[5] Erisoglu, U., Erisoglu, M. and Erol, H. (2011) A Mixture Model of Two Different Distributions Approach to the Analysis of Heterogeneous Survival Data. International Journal of Computational and Mathematical Sciences, 5, 75-79.

[6] Lindeley, D.V. (1958) Fiducial Distributions and Bayes' Theorem. Journal of the Royal Statistical Society, Series B, 20, 102-107. https://doi.org/10.1111/j.2517-6161.1958.tb00278.x

[7] Lindely, D.V. (1965) Introduction to Probability and Statistics from Bayesian Viewpoint, Part II, Inference. Cambridge University Press, New York. 
[8] Ghitany, M.E., Atieh, B. and Nadarajah, S. (2008) Lindley Distribution and Its Applications. Mathematics and Computers in Simulation, 78, 493-506.

https://doi.org/10.1016/j.matcom.2007.06.007

[9] Shanker, R., Sharma, S. and Shanker, R. (2013) A Two-Parameter Lindley Distribution for Modeling Waiting and Survival Times Data. Applied Mathematics, 4, 363-368. https://doi.org/10.4236/am.2013.42056

[10] Shanker, R. and Mishr, A. (2013) A Quasi Lindley Distribution. African Journal of Mathematics and Computer Science Research, 6, 64-71.

[11] Shanker, R. (2015) Shanker Distribution and Its Applications. International Journal of Statistics and Applications, 5, 338-348.

[12] Shanker, R., Shukla, K.K., Shanker, R. and Tekie, A.L. (2017) A Three-Parameter Lindley Distribution. American Journal of Mathematics and Statistics, 7, 15-26.

[13] Monsef, M.M.E.A. (2016) A New Lindley Distribution with Location Parameter. Communications in Statistics- Theory and Methods, 45, 5204-5219. https://doi.org/10.1080/03610926.2014.941496

[14] Shanker, R. (2015) Akash Distribution and Its Applications. International Journal of Probability and Statistics, 4, 65-75.

[15] Renyi, A. (1961) On Measures of Entropy and Information. In: Fourth Berkeley Symposium on Mathematical Statistics and Probability, 547-561.

[16] Shannon, C. and Weaver, W. (1949) The Mathematical Theory of Communication. University of Illinois Press, Chicago, IL.

[17] Patil, G.P. and Rao, G.R. (1978) Weighted Distributions and Size Biased Sampling with Applications to Wildlife Populations and Human Families. Biometrics, 34, 179-189. https://doi.org/10.2307/2530008

[18] Bader, M.G. and Priest, A.M. (1982) Statistical Aspects of Fiberd Le Strength in Hybrid Composites. In: Hayashi, T., et al., Eds., Progress in Science in Engineering Composites, ICCM-IV, Tokyo, Canada, 1129-1136.

[19] Shakil, M., Kibria, B.G. and Singh, J.N. (2010) A New Family of Distributions Based on the Generalized Pearson Di Erential Equation with Some Applications. Austrian Journal of Statistics, 39, 259-278. https://doi.org/10.17713/ajs.v39i3.248

[20] Mol, S., Ozden, O. and Karakulak, S. (2012) Levels of Selected Metals in Albacore (Thunnusalalunga, Bonnaterre, 1788) from the Eastern Mediterranean. Journal of Aquatic Food Product Technology, 21, 111-117. https://doi.org/10.1080/10498850.2011.586489

[21] Fuller, E.R., Freiman, S.W., Quinn, J.B., Quinn, G. and Carter, W. (1994) Fracture Mechanics Approach to the Design of Glass Aircraft Windows: A Case Study. Proceedings of SPIE-The International Society for Optical Engineering, 419-430. https://doi.org/10.1117/12.187363

[22] Nichols, M.D. and Padgett, W.J. (2006) A Bootstrap Control Chart for Weibull Percentiles. Quality and Reliability Engineering International, 22, 141-151. https://doi.org/10.1002/qre.691

[23] Smith, R.L. and Naylor, J.C. (1987) A Comparison of Maximum Likelihood and Bayesian Estimators for the Three Parameter Weibull Distribution. Journal of the Royal Statistical Society, 36, 358-369. https://doi.org/10.2307/2347795

[24] Birnbaum, Z.W. and Saunders, S.C. (1969) Estimation for a Family of Life Distributions with Applications to Fatigue. Journal of Applied Probability, 6, 328-347.

https://doi.org/10.2307/3212004 
[25] Weisberg, S. (2005) Applied Linear Regression. 3rd Edition, Wiley, New York. https://doi.org/10.1002/0471704091

[26] Bjerkedal, T. (1960) Acquisition of Resistance in Guinea Pigs Infected with Different Doses of Virulent Tubercle Bacilli. American Journal of Hygiene, 72, 130-148. https://doi.org/10.1093/oxfordjournals.aje.a120129

[27] Mead, M.E. (2016) On Five-Parameter Lomax Distribution Properties and Applications. Pakistan Journal of Statistics and Operations Research, 1, 185-199. https://doi.org/10.18187/pjsor.v11i4.1163

[28] Choulakian, V. and Stephens, M.A. (2001) Goodness-of-Fit for the Generalized Pareto Distribution. Technometrics, 43, 478-484. https://doi.org/10.1198/00401700152672573

[29] Gross, A.J. and Clark, V.A. (1975) Survival Distributions: Reliability Applications in the Biometrical Sciences. John Wiley, New York. 


\section{Appendix}

\section{Data Sets}

Data set 4 (Mol.et al., (2012)): the data represents the level of mercury [20]. $1.007,1.447,0.763,2.010,1.346,1.243,1.586,0.821,1.735,1.396,1.109$, $0.993,2.007,1.373,2.242,1.647,1.350,0.948,1.501,1.907,1.952,0.996,1.433$, 0.866, 1.049, 1.665,2.139, 0.534, 1.027, 1.678, 1.214, 0.905, 1.525, 0.763 .

Data set 5 (Fuller.et al., (1994): the data represents the strength of glass of the aircraft window [21].

$18.83,20.80,21.657,23.03,23.23,24.05,24.321,25.50,25.52,25.80,26.69$, 26.77, 26.78, 27.05, 27.67, 29.90,31.11, 33.20, 33.73, 33.76, 33.89, 34.76, 35.75, $35.91,36.98,37.08,37.09,39.58,44.045,45.29,45.381$.

Data set 6 (Nichols.et al., (2006): the data are breaking stress of carbon fibers [22].

$3.70,2.74,2.73,2.50,3.60,3.11,3.27,2.87,1.47,3.11,3.56,4.42,2.41,3.19,3.22$, $1.69,3.28,3.09,1.87,3.15,4.90,1.57,2.67,2.93,3.22,3.39,2.81,4.20,3.33,2.55$, $3.31,3.31,2.85,1.25,4.38,1.84,0.39,3.68,2.48,0.85,1.61,2.79,4.70,2.03,1.89$, $2.88,2.82,2.05,3.65,3.75,2.43,2.95,2.97,3.39,2.96,2.35,2.55,2.59,2.03,1.61$, $2.12,3.15,1.08,2.56,1.80,2.53$.

Data set 7 (Smith.et al., (1987): the data represents the strength of glass fibers [23].

$0.55,0.93,1.25,1.36,1.49,1.52,1.58,1.61,1.64,1.68,1.73,1.81,2,0.74,1.04$, $1.27,1.39,1.49,1.53,1.59,1.61,1.66,1.68,1.76,1.82,2.01,0.77,1.11,1.28,1.42$, $1.5,1.54,1.6,1.62,1.66,1.69,1.76,1.84,2.24,0.81,1.13,1.29,1.48,1.5,1.55,1.61$, $1.62,1.66,1.7,1.77,1.84,0.84,1.24,1.3,1.48,1.51,1.55,1.61,1.63,1.67,1.7,1.78$, 1.89 .

Data set 8 (Birnbaum.et al., (1969)): the data are fatigue life of $6061 \mathrm{~T} 6$ aluminum coupons [24].

$70,90,96,97,99,100,103,104,104,105,107,108,108,108,109,109,112,112$, $113,114,114,114,116,119,120,120,120,121,121,123,124,124,124,124,124$, $128,128,129,129,130,130,130,131,131,131,131,131,132,132,132,133,134$, $134,134,134,136,136,137,138,138,138,139,139,141,141,142,142,142,142$, $142,142,144,144,145,146,148,148,149,151,151,152,155,156,157,157,157$, 157, 158, 159,162, 163, 163, 164, 166, 166, 168, 170, 174, 201, 212.

Data set 9 (Weisberg, (2005): the data are athletes' skin folds [25].

28.0, 109.1, 102.8, 104.6, 126.4, 80.3, 75.2, 87.2, 97.9, 75.1, 65.1, 171.1,76.8, $117.8,90.2,97.2,99.9,125.9,69.9,98,96.8,80.3,74.9,83.0,91.0,76.2,52.6$, $111.1,110.7,74.7,113.5,99.8,80.3,109.5,123.6,91.2,49.0,110.2,89.0,98.3,122.1$, 90.4, 106.9,156.6, 101.1, 126.4, 114.0, 70.0, 77.0, 148.9, 80.1, 156.6, 115.9, 181.7, $71.6,143.5,200.8,68.9,103.6,71.3,54.6,88.2,95.4,47.5,55.6,62.9,52.5,62.6$, $49.9,57.9,109.6,98.5,136.3,103.6,102.8,131.9,33.8,43.5,46.2,73.9,36.8,67$, 41.1, 59.4, 48.4, 50.0, 54.6, 42.3, 46.1, 46.3, 109.0,98.1, 80.6, 68.3, 47.6, 61.9, 38.2, $43.5,56.8,41.6,58.9,44.5,41.8,33.7,50.9,40.5,51.2,54.4,52.3,57.0,65.3,52.0$, $42.7,35.2,49.2,61.8,46.5,34.8,60.2,48.1,44.5,54.0,44.7,64.9,43.8,58.3,52.8$, 
$43.1,78.0,40.8,41.5,50.9,49.6,88.9,48.3,61.8,43.0,61.1,43.8,54.2,41.8$, $34.1,30.5,34.0,46.7,71.1,65.9,34.3,34.6,31.8,34.5,31.0,32.6,31.5,32.6,31.0$, $33.7,30.3,38.0,55.7,37.5,112.5,82.7,29.7,38.9,44.8,30.9,44.0,37.5,37.6,31.7$, $36.6,48,41.9,30.9,52.8,43.2,113.5,96.9,49.3,42.3,96.3,56.5,105.7,100.7,56.8$, $75.9,52.8,47.8,76.0,61.2,75.6,43.3,49.5,70.0,75.7,57.7,67.2,56.5,47.6,60.4$, 34.9.

Data set 10 (Bjerkedal, (1960)): the data represents he survival times of guinea pigs [26].

$0.1,0.33,0.44,0.56,0.59,0.72,0.74,0.77,0.92,0.93,0.96,1,1,1.02,1.05,1.07$, $1.07,1.08,1.08,1.08,1.09,1.12,1.13,1.15,1.16,1.2,1.21,1.22,1.22,1.24,1.3$, $1.34,1.36,1.39,1.44,1.46,1.53,1.59,1.6,1.63,1.63,1.68,1.71,1.72,1.76,1.83$, $1.95,1.96,1.97,2.02,2.13,2.15,2.16,2.22,2.3,2.31,2.4,2.45,2.51,2.53,2.54$, $2.54,2.78,2.93,3.27,3.42,3.47,3.61,4.02,4.32,4.58,5.55$.

Data set 11 (Mead, (2016)): the data consist the taxes revenue [27].

$5.9,20.4,14.9,16.2,17.2,7.8,6.1,9.2,10.2,9.6,13.3,8.5,21.6,18.5,5.1,6.7,17$, 8.6, 9.7, 39.2, 35.7, 15.7, 9.7, 10, 4.1, 36, 8.5, 8, 9.2, 26.2,21.9,16.7, 21.3, 35.4, 14.3, $8.5,10.6,19.1,20.5,7.1,7.7,18.1,16.5,11.9,7,8.6,12.5,10.3,11.2,6.1,8.4,11$, $11.6,11.9,5.2,6.8,8.9,7.1,10.8$.

Data set 12 (Choulakian.et al., (2001): the data are flood peaks [28].

$1.7,2.2,14.4,1.1,0.4,20.6,5.3,0.7,1.9,13.0,12.0,9.3,1.4,18.7,8.5,25.5,11.6$, $14.1,22.1,1.1,2.5,27.0,14.4,1.7,37.6,0.6,2.2,39.0,0.3,15.0,11.0,7.3,22.9,1.7$, $0.1,1.1,0.6,9.0,1.7,7.0,20.1,0.4,2.8,14.1,9.9,10.4,10.7,30.0,3.6,5.6,30.8$, $13.3,4.2,25.5,3.4,11.9,21.5,27.6,36.4,2.7,64.0,1.5,2.5,27.4,1.0,27.1,20.2,16.8$, $5.3,9.7,27.5,2.5$.

Data set 13 (Gross.et al., (1975)): the data represent the relief times of patients [29].

$1.1,1.4,1.3,1.7,1.9,1.8,1.6,2.2,1.7,2.7,4.1,1.8,1.5,1.2,1.4,3.0,1.7,2.3,1.6$, 2.0 . 
Table S1. MLEs, AIC, BIC, K-S statistics and its critical value of the fitted distributions.

\begin{tabular}{|c|c|c|c|c|c|c|c|}
\hline Data & Model & MLE & $-2 \log L$ & AIC & BIC & K-S Statistic & Critical value \\
\hline \multirow{16}{*}{ Data 1} & NGAD & $\hat{\theta}=2.428$ & 117.756 & 123.756 & 130.458 & 0.138 & \\
\hline & & $\hat{\beta}=1.216$ & & & & & \\
\hline & & $\hat{\eta}=5.308 \times 10^{5}$ & & & & & \\
\hline & LwLD & $\hat{\theta}=1.695$ & 128.735 & 134.735 & 141.437 & 0.185 & \\
\hline & & $\hat{\beta}=1.272$ & & & & & \\
\hline & & $\hat{\alpha}=8442.147$ & & & & & \\
\hline & TwPLD & $\hat{\theta}=0.408$ & 261.735 & 265.735 & 270.203 & 0.434 & 0.164 \\
\hline & & $\hat{\alpha}=-3.065 \times 10^{-17}$ & & & & & \\
\hline & QLD & $\hat{\theta}=0.408$ & 261.735 & 265.735 & 270.203 & 0.434 & \\
\hline & & $\hat{\alpha}=12.274 \times 10^{3}$ & & & & & \\
\hline & ThPLD & $\hat{\theta}=0.408$ & 263.7358 & 267.735 & 274.438 & 0.434 & \\
\hline & & $\hat{\beta}=1.716 \times 10^{-4}$ & & & & & \\
\hline & & $\hat{\alpha}=8.151 \times 10^{4}$ & & & & & \\
\hline & $\mathrm{AD}$ & $\hat{\theta}=0.965$ & 224.280 & 226.280 & 232.982 & 0.348 & \\
\hline & $\mathrm{LD}$ & $\hat{\theta}=0.651$ & 233.021 & 235.021 & 237.255 & 0.349 & \\
\hline & SD & $\hat{\theta}=0.654$ & 238.381 & 240.381 & 242.615 & 0.387 & \\
\hline \multirow{16}{*}{ Data 2} & NGAD & $\hat{\theta}=0.047$ & 225.825 & 231.825 & 235.232 & 0.111 & \\
\hline & & $\hat{\beta}=8.326$ & & & & & \\
\hline & & $\hat{\eta}=5.178 \times 10^{3}$ & & & & & \\
\hline & LwLD & $\hat{\theta}=0.034$ & 226.789 & 232.209 & 235.616 & 0.090 & \\
\hline & & $\hat{\beta}=14.281$ & & & & & \\
\hline & & $\hat{\alpha}=239.790$ & & & & & \\
\hline & TwPLD & $\hat{\theta}=0.0138$ & 242.873 & 246.873 & 249.144 & 0.263 & 0.283 \\
\hline & & $\hat{\alpha}=1.635 \times 10^{-14}$ & & & & & \\
\hline & QLD & $\hat{\theta}=0.014$ & 242.873 & 246.873 & 249.144 & 0.263 & \\
\hline & & $\hat{\alpha}=1.635 \times 10^{-14}$ & & & & & \\
\hline & ThPLD & $\hat{\theta}=0.408$ & 242.873 & 248.873 & 252.279 & 0.263 & \\
\hline & & $\hat{\beta}=15.372$ & & & & & \\
\hline & & $\hat{\alpha}=-2.652 \times 10^{-15}$ & & & & & \\
\hline & $\mathrm{AD}$ & $\hat{\theta}=0.042$ & 227.058 & 229.058 & 232.465 & 0.107 & \\
\hline & $\mathrm{LD}$ & $\hat{\theta}=0.027$ & 231.471 & 233.471 & 234.606 & 0.149 & \\
\hline & SD & $\hat{\theta}=0.028$ & 231.060 & 233.060 & 234.196 & 0.145 & \\
\hline \multirow{6}{*}{ Data 3} & NGAD & $\hat{\theta}=0.814$ & 83.992 & 89.992 & 92.979 & 0.171 & \\
\hline & & $\hat{\beta}=0.853$ & & & & & \\
\hline & & $\hat{\eta}=2.664 \times 10^{5}$ & & & & & \\
\hline & LwLD & $\hat{\theta}=0.629$ & 82.891 & 88.891 & 91.878 & 0.166 & \\
\hline & & $\hat{\beta}=1.358$ & & & & & \\
\hline & & $\hat{\alpha}=4.561 \times 10^{5}$ & & & & & \\
\hline
\end{tabular}




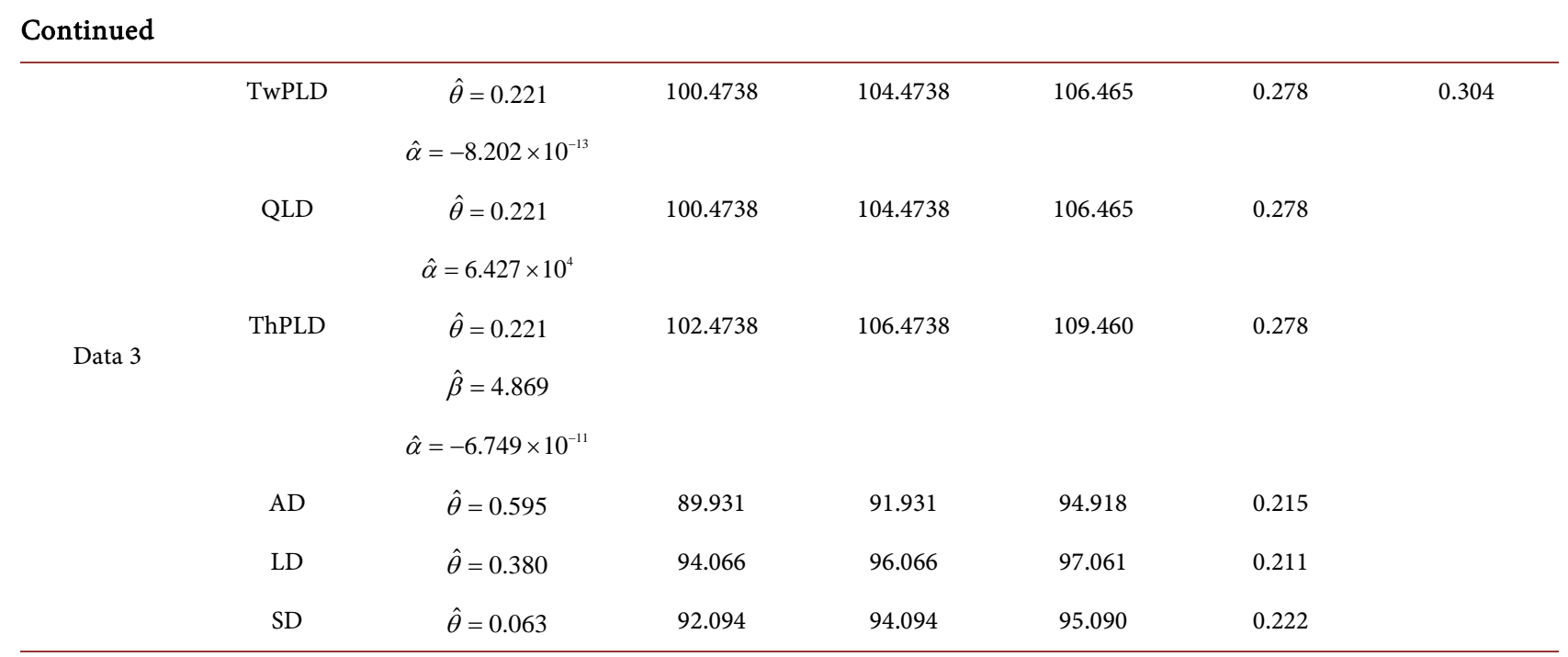

